\title{
Cardiomegalia gigante
}

Luisa María Charco-Roca, María Garví-López y Lorena Moreno-De la Rosa

Universidad de Castilla-La Mancha, Hospital General Universitario de Albacete; Centro de Salud de Villanueva de la Jara. Castilla-La Mancha, España

\section{Resumen}

Mujer de 80 años con antecedentes de hipertensión arterial, valvulopatía reumática con recambio valvular mitral y aórtica, fibrilación auricular crónica e insuficiencia cardiaca congestiva dependiente de oxígeno crónico domiciliario durante 17 horas diarias. El motivo de llegada al hospital fue ictus cerebral de etiología cardioembólica.

PALABRAS CLAVE: Cardiomegalia. Dilatación biauricular. Radiografía de tórax.

\begin{abstract}
Eighty-year old woman with a medical history of arterial hypertension, rheumatic valvular heart disease with mitral and aortic valve replacement, chronic atrial fibrillation and congestive heart failure dependent on domiciliary chronic oxygen for 17 daily hours. The reason for arrival to the hospital was a stroke of cardioembolic etiology.
\end{abstract}

KEY WORDS: Cardiomegaly. Biatrial enlargement. Chest radiograph.

Mujer de 80 años con antecedentes de hipertensión arterial, valvulopatía reumática con recambio valvular mitral y aórtica, fibrilación auricular crónica e insuficiencia cardiaca congestiva dependiente de oxígeno crónico domiciliario durante 17 horas diarias. El motivo de llegada al hospital fue ictus cerebral de etiología cardioembólica. En la radiografía de tórax se observó cardiomegalia con horizontalización de la bifurcación traqueal (Figura 1), signo indirecto de dilatación de las cavidades cardiacas y que puede ayudar al diagnóstico diferencial con el derrame pericárdico.

La ecocardiografía de la paciente mostraba dilatación severa de ambas aurículas y dilatación ventricular derecha con fracción de eyección ventricular derecha severamente disminuida, así como fracción de eyección ventricular izquierda conservada. La dilatación de ambas aurículas se puede observar en el electrocardiograma como aumento del voltaje y duración de la onda $\mathrm{P}$ (mayor de $2.5 \mathrm{mV}$ o $\mathrm{P}$ pulmonale), sobre todo en $\mathrm{V} 1$ y derivaciones inferiores.

El aumento de ambas cavidades auriculares puede presentarse como una causa inusual de cardiomegalia masiva. Las causas del agrandamiento biauricular incluyen cardiomiopatía restrictiva, enfermedad cardiaca reumática, insuficiencia mitral aislada y pericarditis constrictiva. ${ }^{1}$ Las consecuencias clínicas de la dilatación biauricular severa se derivan de la insuficiencia cardiaca congestiva y de la compresión de estructuras adyacentes; la manifestación más frecuente es la disnea. ${ }^{2}$

En la mayoría de los casos, una radiografía de tórax será suficiente para detectar la cardiomegalia. La ampliación específica de las cámaras cardiacas puede
Gac Med Mex. 2018;154:727-728 


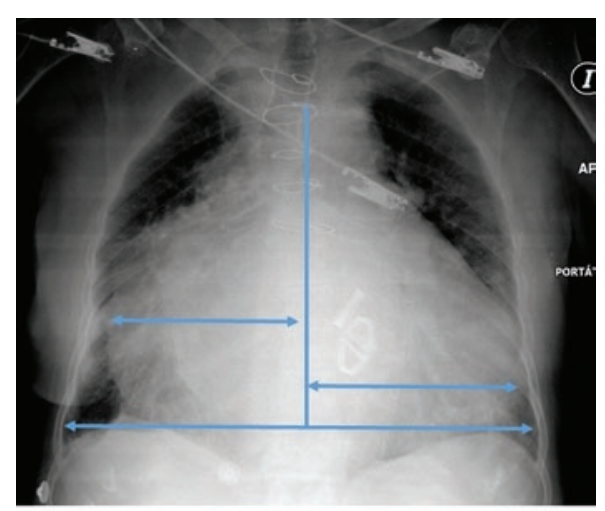

Indice cardiotorácico

$$
\frac{a+b}{c}
$$

Figura 1. Radiografía de tórax portátil anteroposterior. Índice cardiotorácico de 0.8 . reconocerse por los cambios en el contorno cardiaco, interfaces nuevas o diferentes con el pulmón adyacente 0 el desplazamiento de las estructuras mediastínicas adyacentes. La relación cardiotorácica o índice cardiotorácico es la relación entre el diámetro cardiaco horizontal máximo y el diámetro torácico horizontal máximo (Figura 1). El índice cardiotorácico supone un cálculo sencillo que ayuda a detectar la ampliación de la silueta cardiaca y es considerado normal cuando es menor o igual a 0.5 .

\section{Bibliografía}

1. Sethi $T$, Singh AP, Singla $V$, Singh $Y$. Biatrial enlargement: an unusual cause of massive cardiomegaly. BMJ Case Reports. 2013;2013.

2. Rogers WR, Wittels B. extreme bilateral atriomegaly; review of the literature and report of a case. Circulation. 1957;15:434-441. 